\title{
Polymer Brushes-Induced Hollow Colloids via Diffusion-Controlled Silication
}

Lei Tian, ${ }^{1,2,+*}$ Bei Li, ${ }^{1}$ Qiuyu Zhang, ${ }^{1 *}$ and Yufeng Wang $^{2 *}$

${ }^{1}$ Department of Applied Chemistry, School of Natural and Applied Sciences, Northwestern Polytechnical University, Xi' an 710072, China

${ }^{2}$ Department of Chemistry, The University of Hong Kong, Pokfulam Road, Hong Kong SAR, China

*Corresponding author:

(Lei Tian). E-mail: leitian@szu.edu.cn,

(Qiuyu Zhang). E-mail: qyzhang@nwpu.edu.cn,

(Yufeng Wang). E-mail: wanglab@hku.hk

Number of pages: 5

Number of figures: 4

List of contents

Figure S1. SEM image of PGMA particles synthesized by dispersion polymerization.

Figure S2. SEM images of hollow particles treated by DMF (a, a') and THF (b, b') for 12 h. TEM images of hollow particles treated by THF (c, c').

Figure S3. h/R values of hollow colloids with different ATRP times.

Figure S4. Structural diversity of Janus hollow structures. 


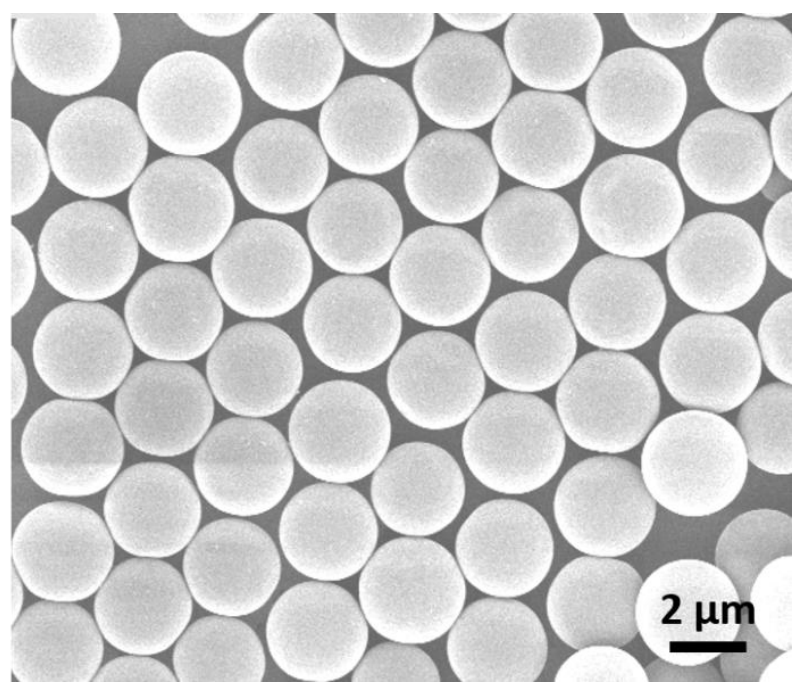

Figure S1. SEM image of PGMA particles synthesized by dispersion polymerization. 

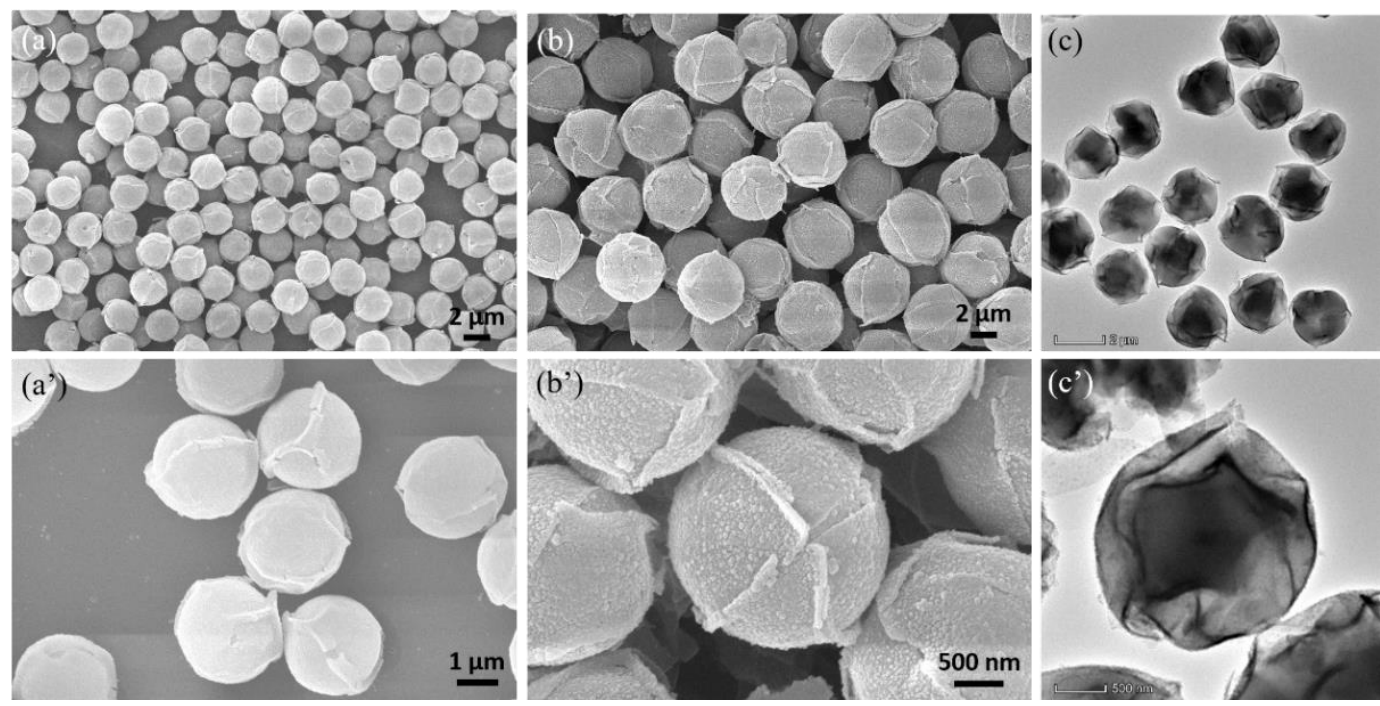

Figure S2. SEM images of hollow particles treated by DMF (a, a') and THF (b, b') for $12 \mathrm{~h}$. TEM images of hollow particles treated by THF (c, c'). 


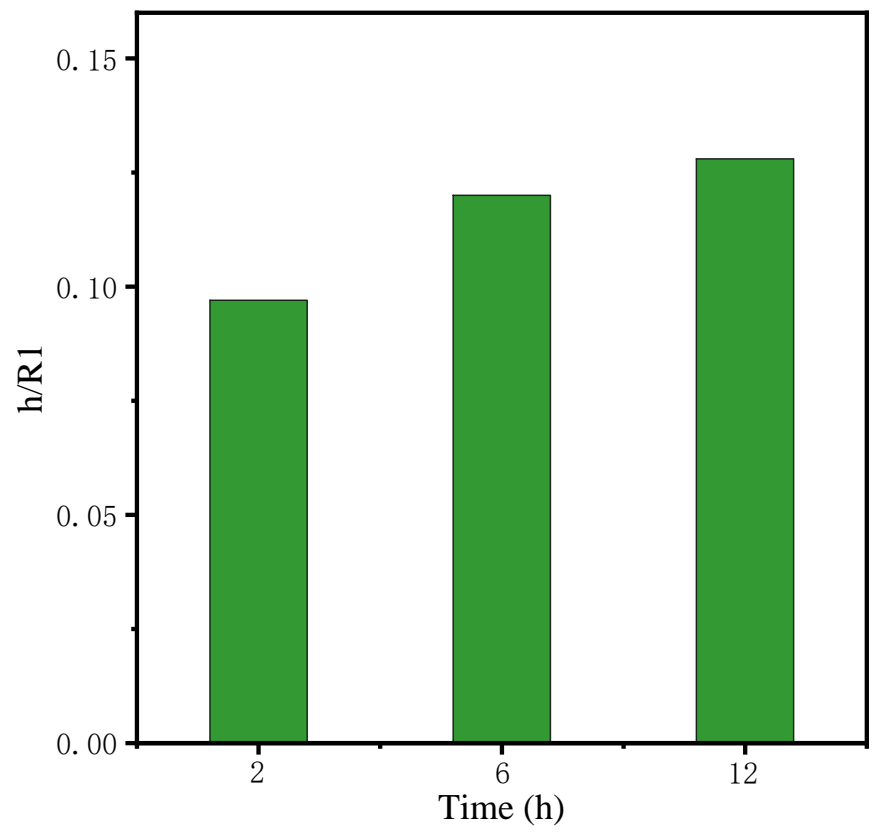

Figure S3. h/R values of hollow colloids with different ATRP times. 


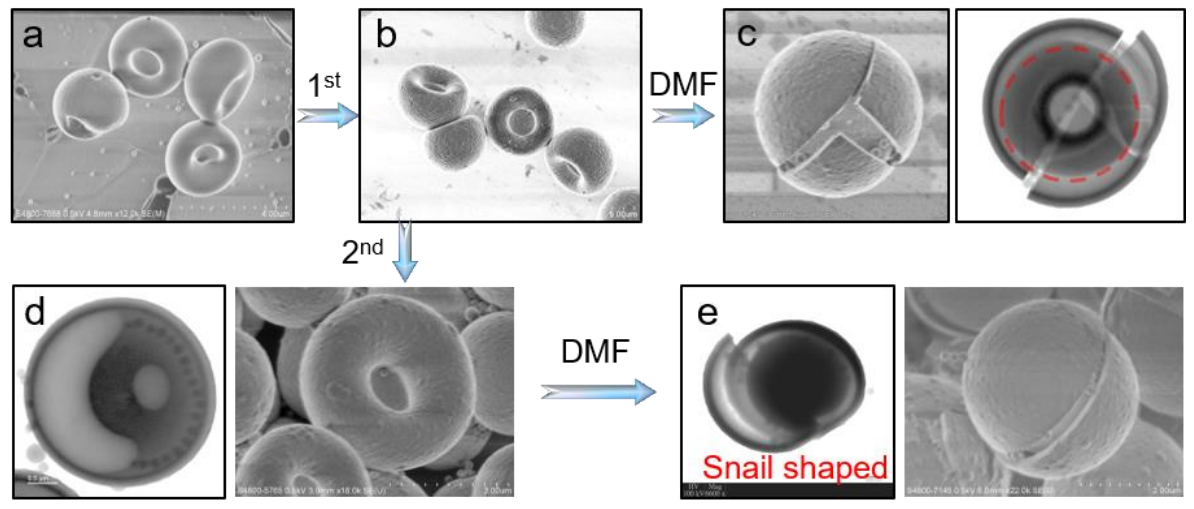

Figure S4. Structural diversity of Janus hollow structures. a, Bowl-shaped PGMA particles are fabricated. $b$, The particles remain the bowl shape after $1^{\text {st }}$ silication. $c$, Spherical hollow structures are formed via the treatment of DMF but with intercavity. d, TEM and SEM of bowl-like hollow structures by $2^{\text {nd }}$ silication. After DMF treatment, snail-shaped spheres are also synthesized (e). 